\title{
American College of Sports Medicine
}

\author{
Posicionamento Oficial
}

\section{Exercício para pacientes com doença arterial coronariana}

\section{RESUMO}

O treinamento físico aumenta a capacidade funcional e reduz os sintomas nos pacientes coronariopatas. Entretanto, tais pacientes têm um risco maior de complicações durante a prática de exercícios; portanto, devem-se seguir orientações adequadas para minimizar esses riscos. Com base nos benefícios e nos riscos documentados do exercício físico para pacientes com doença arterial coronariana, é Posicionamento Oficial do Colégio Americano de Medicina do Esporte que a maior parte dos pacientes coronariopatas deve participar de um programa de exercícios individualmente prescritos para atingir uma saúde ideal dos pontos de vista físico e emocional.

\section{INTRODUÇÃO}

Este Posicionamento Oficial tratará do exercício para pacientes com doença arterial coronariana (DAC). Os pontos seguintes são prontamente reconhecidos. Os pacientes com DAC não são um grupo homogêneo e devem ser analisadas individualmente. Eles variam muito em seu estado clínico, o que inclui: gravidade da doença coronariana, grau de disfunção ventricular esquerda, potencial para desenvolver isquemia miocárdica e presença de arritmias cardíacas. Alguns pacientes coronariopatas tiveram previamente eventos cardíacos maiores (p.ex., infarto do miocárdio ou parada cardiorrespiratória) ou foram submetidos a tratamentos invasivos (p.ex., cirurgia de revascularização miocárdica, angioplastia coronariana, etc.). Muitos pacientes são portadores de outras doenças, como hipertensão, doença vascular periférica, doença valvar, doença pulmonar obstrutiva crônica e diabetes mellitus.

Este Posicionamento Oficial abordará o exercício para o paciente não internado, embora uma deambulação precoce para pacientes internados após eventos cardíacos maiores também seja importante.

Traduzido do original Exercise for patients with coronary artery disease. Med Sci Sports Exerc 1994;26:i-v.

\section{EFEITOS DO TREINAMENTO FÍSICO}

\section{Capacidade funcional}

Os pacientes com DAC em geral têm menor consumo máximo de oxigênio e menor tolerância ao exercício do que seus pares saudáveis. A magnitude dessa redução varia em parte com a gravidade da doença e alguns pacientes coronariopatas têm tolerância normal ao esforço. Menor volume sistólico máximo e menor frequiência cardíaca máxima podem limitar o débito cardíaco máximo e o consumo de oxigênio ${ }^{1}$. A magnitude da redução do volume sistólico depende da quantidade de miocárdio que apresenta sofrimento isquêmico com o exercício e/ou do tamanho do infarto do miocárdio prévio. $\mathrm{O}$ mecanismo para a menor freqüência cardíaca com o exercício em pacientes não medicados ainda não está bem definido. O desempenho máximo com o exercício em pacientes com angina de peito é limitado pelo desconforto. Em pacientes com angina clássica, tal desconforto ocorre em duplo produto (freqüência cardíaca vezes pressão arterial sistólica) altamente reprodutível ${ }^{2}$, desde que fatores como hora do dia, temperatura ambiente e posição corporal sejam constantes ${ }^{3}$. Muitos pacientes não demonstram o padrão clássico, sugerindo que alterações coronarianas por vasoespasmo contribuam para essa variação em seu limiar anginoso ${ }^{4}$.

Tanto o comportamento do paciente quanto as recomendações médicas podem também reduzir a capacidade funcional. O destreinamento ocorre por restrições de atividade física autoinduzida e iatrogênicas. Medicamentos como betabloqueadores, embora sejam benéficos para pacientes com sintomas, podem reduzir a capacidade funcional em alguns grupos de pacientes, principalmente se essas drogas forem prescritas rotineiramente ou profilaticamente em pacientes assintomáticos.

O treinamento físico aumenta a capacidade funcional e o consumo máximo de oxigênio $\left(\dot{\mathrm{V}}_{2 \text { máx }}\right)$ em pacientes coronariopatas, aumentando a diferença arteriovenosa de oxigênio e em alguns casos também por aumento do volume sistólico máximo ${ }^{1}$. A contribuição relativa desses dois fatores para o aumento do $\dot{\mathrm{VO}}_{2 \text { máx }}$ varia com as características do paciente e com o tipo de programa de treinamento. $\mathrm{O}$ aumento do 
$\dot{\mathrm{V}} \mathrm{O}_{2 \text { máx }}$ nos pacientes coronariopatas após três meses de treinamento é de aproximadamente 10 a $60 \%$ nos relatos publicados, ficando em média em $20 \%^{5,6}$. Esses aumentos da capacidade funcional podem subestimar os benéficos funcionais do exercício regular, porque aumentos importantes da capacidade de endurance submáxima podem ocorrer em indivíduos saudáveis com aumentos apenas modestos do $\dot{\mathrm{V}} \mathrm{O}_{2 \operatorname{máx}}{ }^{7}$.

\section{Sintomas de isquemia miocárdica}

Algumas das principais melhoras da tolerância aos esforços obtidas com o exercício regular ocorrem em pacientes com angina ${ }^{8}$. O treinamento reduz a frequiência cardíaca submáxima para qualquer carga de trabalho ou atividade e retarda o surgimento de sintomas durante o exercício. Alguns pacientes apresentam de fato desaparecimento dos sintomas anginosos com o treinamento ${ }^{8}$. Essa redução dos sintomas anginosos obtida com o exercício regular pode facilitar uma redução das doses dos medicamentos, mas este benefício do exercício ainda não foi bem quantificado.

\section{Isquemia e perfusão miocárdica}

Apesar do melhor desempenho físico e da redução dos sintomas nos pacientes coronariopatas, não há evidências conclusivas de que o treinamento físico isoladamente possa aumentar o calibre dos vasos, aumentar o desenvolvimento de colaterais ou reverter as obstruções coronarianas ${ }^{6,9}$. Foram documentados aumentos do diâmetro coronariano com o treinamento em modelos animais de aterosclerose induzida por dieta ${ }^{10}$. Além disso, alguns estudos envolvendo treinamento físico observaram aumentos do duplo produto necessário para desencadear isquemia e menores infradesníveis do segmento ST em duplos produtos semelhantes, indicando melhor fluxo coronariano ${ }^{11}$, mas essas melhoras não foram encontradas universalmente. A cintilografia miocárdica com tálio-201 documentou melhor perfusão miocárdica em alguns pacientes após o treinamento ${ }^{12}$. Nesses estudos não foi avaliado o papel da redução das obstruções coronarianas e estudos angiográficos não conseguiram demonstrar alterações do calibre das coronárias ou dos vasos colaterais em repouso ${ }^{6}$. Conseqüentemente, embora a perfusão coronariana seja melhor em alguns pacientes após o treinamento físico, o mecanismo ainda permanece indefinido?.

\section{Fatores de risco para doença arterial coronariana}

Perfil lipídico. Níveis aumentados de colesterol de baixa densidade (LDL) e níveis reduzidos de colesterol de alta densidade (HDL) são fatores de risco importantes para o desenvolvimento de doença arterial obstrutiva coronariana. Estudos recentes também demonstram a importância da modificação desse perfil lipídico na prevenção secundária da doença coronariana ${ }^{13}$. Uma meta-análise dos oito estudos clínicos de redução do colesterol em sobreviventes de um infarto do mio- cárdio entre 1965 e 1988 demonstra redução de $16 \%$ de reinfartos fatais e $25 \%$ de reinfartos não fatais no grupo tratado ${ }^{14}$. $\mathrm{O}$ tratamento desse perfil lipídico envolveu somente dieta em três estudos e dieta mais medicamentos nos cinco restantes. Nenhum desses estudos, no entanto, envolveu diretamente um programa de reabilitação cardíaca, nem - com raras exceções ${ }^{15}$ - os programas de exercícios analisaram a relação entre modificações no perfil lipídico e sobrevivência. Uma meta-análise de 15 estudos sobre o efeito do treinamento físico em pacientes pós-infarto agudo do miocárdio mostrou reduções importantes do colesterol total, do colesterol LDL e dos triglicerídeos; e aumento do colesterol HDL com o treinamento ${ }^{16}$. Esses resultados sugerem que programas bem estruturados de reabilitação cardíaca que utilizam exercício, dieta e medicamentos, quando adequado, alteram de maneira benéfica o perfil lipídico e o prognóstico dos pacientes.

Tabagismo. O tabagismo é reconhecido como um fator de risco importante para doença coronariana, particularmente morte súbita. Além disso, homens que sobrevivem a um infarto do miocárdio e param de fumar têm taxa de mortalidade de $19 \%$ nos seis anos seguintes, enquanto a mortalidade naqueles que continuam a fumar é de $30 \%{ }^{17}$. Um estudo sobre os efeitos do treinamento sobre o hábito de fumar em pacientes convalescentes de um infarto agudo do miocárdio sugerem que os programas formais de reabilitação cardíaca facilitam o ato de parar de fumar e sua manutenção ${ }^{18}$, mas não está disponível um suporte sólido para esta conclusão.

Hipertensão arterial. A hipertensão arterial não controlada dobra ou triplica o risco de eventos cardiovasculares e a pressão alta é um fator preditor independente de subseqüente morbidade e mortalidade em sobrevivência de um infarto do miocárdio ${ }^{19}$. Um controle efetivo da pressão arterial em indivíduos pós-infarto reduz a mortalidade cardiovascular em $20 \%{ }^{20}$. O treinamento pode contribuir para o controle da pressão arterial $^{21}$, mas um controle ideal da pressão arterial é habitualmente atingido com tratamento farmacológico. $\mathrm{O}$ treinamento pode ainda contribuir para o controle da pressão arterial indiretamente através da redução ponderal, mas sua contribuição independente para o controle da pressão arterial em pacientes com doença coronariana ainda não foi bem documentada.

Intolerância à glicose e "diabetes mellitus". A intolerância à glicose e o diabetes mellitus são importantes fatores de risco para doenças cardiovasculares. Infelizmente, não se demonstrou que o controle do diabetes influencie beneficamente os pacientes coronariopatas. A atividade física pode auxiliar no controle da hiperglicemia, principalmente quando combinado com a redução ponderal ${ }^{22}$. A atividade física é benéfica para o controle da glicemia em pacientes coronariopatas.

Controle da obesidade. A obesidade é um fator de risco independente para o desenvolvimento de doença coronaria$\mathrm{na}^{23}$. A obesidade está também associada com hipertensão, 
intolerância à glicose e perfis lipídicos alterados ${ }^{24}$. A redução ponderal é um benefício obtido em programas de exercícios e contribui para menor morbidade e mortalidade cardiovasculares ${ }^{15,25,26}$.

Benefícios psicológicos. Estudos demonstraram que pacientes submetidos a um programa de exercícios ou a um programa de exercícios e aconselhamento psicológico tiveram melhor qualidade de vida, em comparação com grupos-contro$\mathrm{le}^{27}$. Além disso, foi documentado que o treinamento físico reduz a depressão em pacientes pós-infarto ${ }^{28}$. Tais benefícios psicológicos podem representar um benefício importante em programas de reabilitação, mas dois estudos recentes não conseguiram documentar estes benefícios ${ }^{29,30}$

Mortalidade cardiovascular. Estudos publicados documentaram os efeitos benéficos dos programas de reabilitação cardíaca na redução da mortalidade cardiovascular ${ }^{31,32}$. Em comparação com grupos-controle, os pacientes que aderiram a um programa de reabilitação cardíaca tiveram redução de 20 a $25 \%$ nos eventos cardiovasculares fatais e na mortalidade total. Essas análises não mostraram redução nos eventos não fatais. Ainda, não foi avaliada a contribuição do treinamento físico sobre a sobrevivência dos pacientes coronariopatas após cirurgia de revascularização miocárdica ou angioplastia. Logo, os resultados mostram que o exercício físico regular é uma das poucas intervenções que comprovadamente aumentam a sobrevivência após um infarto do miocárdio.

Relação custo/benefício. A relação custo/benefício da reabilitação cardíaca em pacientes pós-infarto do miocárdio ou submetidos a cirurgia de revascularização miocárdica não foi bem estudada. Entretanto, foi relatada redução importante nos custos médicos de pacientes que optaram por participar de programas de reabilitação cardíaca em comparação com os que não participaram ${ }^{33}$. Em outro estudo, pacientes submetidos a reabilitação cardíaca após angioplastia coronariana tiveram menos reinternações e redução nas despesas médicas em comparação com os não submetidos à reabilitação ${ }^{34}$. Esses resultados preliminares sugerem que os benefícios financeiros da reabilitação cardíaca são superiores ao seu custo monetário.

\section{RECOMENDAÇÕES}

\section{Avaliação}

Antes de iniciar um programa de exercícios, pacientes com doença arterial coronariana necessitam de uma história clínica completa, um exame físico detalhado e um teste de esforço máximo ${ }^{35,36}$. A avaliação inicial é focalizada no estado cardiovascular do paciente, bem como no estado clínico geral e ortopédico. Uma avaliação adicional, se clinicamente indicada, é dirigida para a definição de anormalidades fisiopatológicas, incluindo disfunção ventricular esquerda, isquemia miocárdica ou arritmias cardíacas. Anormalidades identificadas podem então ser tratadas clinicamente ou cirurgicamente antes de iniciar o programa de exercícios.

Pacientes identificados como de alto risco para complicações cardiovasculares durante o exercício incluem aqueles com angina instável, estenose aórtica grave, arritmias cardíacas não controladas, insuficiência cardíaca congestiva descompensada ou outras condições clínicas que possam ser agravadas pelo exercício (p.ex., miocardite aguda ou doenças infecciosas) ${ }^{36}$. Esses pacientes devem adiar o treinamento físico até que suas condições estejam estabilizadas.

Pacientes com maior risco que podem exercitar-se sob supervisão médica direta incluem aqueles $\operatorname{com}^{36-38}$ : i) função ventricular esquerda importantemente comprometida; ii) arritmias ventriculares complexas em repouso; iii) arritmias ventriculares que surgem ou pioram com o exercício; iv) redução da pressão arterial sistólica com o exercício; v) sobreviventes de morte súbita cardíaca; vi) infarto do miocárdio recente complicado com insuficiência cardíaca congestiva; e vii) isquemia importante induzida pelo esforço. Deve-se enfatizar, no entanto, que a relação risco/benefício do treinamento para esses pacientes ainda não foi definida.

A prescrição de exercícios, especialmente em termos de intensidade e grau de monitorização e supervisão, é também baseada na avaliação clínica e funcional inicial.

Uma reavaliação deve ser realizada periodicamente ou se houver indicação clínica. Dá-se geralmente dois ou três meses após o início do programa, e a partir daí pelo menos anualmente $^{36}$. É importante avaliar as modificações fisiológicas resultantes de um programa de exercícios, além da possibilidade de progressão da doença.

\section{Prescrição de exercício}

O exercício para os pacientes coronariopatas inclui atividades realizadas em programas formais de exercício e também atividades do cotidiano. Portanto, atividades gerais do dia-a-dia são estimuladas, além das sessões formais de exercícios.

O programa de exercícios para o paciente coronariopata baseia-se na prescrição tradicional para obter efeito de treinamento em indivíduos saudáveis ${ }^{39}$. Entretanto, sofre modificações de acordo com o estado clínico geral e cardiovascular do paciente. Envolve um programa adequado individualmente em termos de tipos de exercícios, bem como freqüência, duração, intensidade e progressão $0^{35,36,39}$.

Tipo. Exercícios contínuos, envolvendo grandes grupos musculares, como caminhada, jogging, ciclismo, natação, ginástica aeróbica e remo, são adequados para condicionamento cardiovascular de endurance. Exercícios de membros superiores com ergômetros especiais podem também ser empregados em indivíduos que não tolerem exercícios de membros inferiores por razões ortopédicas ou outras e naqueles cujas atividades profissionais ou de lazer envolvam predominantemente trabalho de membros superiores. $\mathrm{O}$ treinamento 
de força também é benéfico para pacientes selecionados ${ }^{40}$. Exercícios contra resistência são habitualmente realizados utilizando um esquema de treinamento em circuito, com até 10 a 12 exercícios utilizando 10 a 12 repetições com resistência que permita execução confortável ${ }^{41}$. $\mathrm{O}$ cross-training também pode reduzir problemas ortopédicos e aumentar a aderência.

Freqüência. A freqüência mínima é de três dias não consecutivos por semana. Alguns pacientes preferem exercitar-se diariamente. Contudo, à medida que a freqüência aumenta, aumenta também o risco de lesões músculo-esqueléticas ${ }^{42}$.

Duração. Períodos de aquecimento e volta à calma de pelo menos 10 minutos, incluindo exercícios de alongamento e flexibilidade, devem vir antes e depois de uma sessão de 20 a 40 minutos de duração envolvendo exercícios cardiovasculares realizados continuamente ou através de treinamento intervalado. Este último tipo pode ser especialmente útil para pacientes com doença vascular periférica e claudicação intermitente.

Intensidade. $\mathrm{O}$ exercício em programas supervisionados é realizado a uma intensidade moderada e confortável, geralmente entre 40 e $85 \%$ da capacidade funcional máxima $\left(\dot{\mathrm{V}}_{2 \text { máx }}\right)$, que corresponde a 40 a $85 \%$ da reserva de freqüência cardíaca (FC) máxima ([FC máxima - FC de repouso] $\mathrm{x}$ $40-85 \%$ + FC de repouso), ou 55 a $90 \%$ da FC máxima. O índice de percepção de esforço (IPE), ou escala de Borg, pode também ser utilizado para monitorizar a intensidade do exercício, com o objetivo de mantê-la em nível moderado. A intensidade do esforço deve ser abaixo do nível que provoque isquemia miocárdica, arritmias importantes ou sintomas de intolerância ao esforço, conforme a avaliação prévia pelo teste de esforço.

A intensidade recomendada do treinamento varia com o grau de supervisão disponível e o nível de risco do paciente. Intensidades mais baixas são indicadas para pacientes de alto risco (definidos acima), especialmente quando se exercitam fora de programas supervisionados ou sem monitorização eletrocardiográfica contínua.

Progressão. Qualquer programa de exercícios para pacientes coronariopatas deve envolver progressão inicial lenta e gradual da duração e intensidade.

\section{Supervisão e monitorização}

A supervisão dos pacientes envolve a observação direta do paciente e monitorização da freqüência e do ritmo cardíaco. A medida da pressão arterial é geralmente feita quando há indicação. A natureza e o grau de supervisão e monitorização dependem do risco do paciente de apresentar complicações com o exercício e também da intensidade executada. A supervisão e a monitorização devem ser mais rigorosas quando se lida com pacientes de alto risco (definidos acima). Os pacientes que se exercitam sem supervisão médica e sem monitorização devem fazê-lo em baixas intensidades.
Riscos do exercício. As principais complicações cardiovasculares durante o exercício em pacientes coronariopatas são o infarto agudo do miocárdio, a parada cardiorrespiratória e a morte súbita. A incidência estimada de complicações cardiovasculares em programas supervisionados de reabilitação cardíaca são: um infarto agudo do miocárdio para cada 294.000 pacientes-hora, uma parada cardiorrespiratória para cada 112.000 pacientes-hora e uma morte para cada 784.000 pacientes-hora ${ }^{43}$. Mais de $80 \%$ dos pacientes que sofreram uma parada cardiorrespiratória (em geral por fibrilação ventricular ou taquicardia ventricular) em programas supervisionados de reabilitação cardíaca foram ressuscitados com sucesso com desfibrilação imediata ${ }^{43}$.

\section{CONCLUSÃO}

É posição do Colégio Americano de Medicina do Esporte que a maior parte dos pacientes portadores de doença arterial coronariana deve participar de programas de exercícios individualizados para atingir uma saúde física e mental ideal. Recomenda-se que tais programas incluam uma avaliação médica prévia completa, incluindo um teste de esforço máximo, e uma prescrição individualizada de exercícios.

Programas adequados de exercícios para pacientes coronariopatas oferecem vários benefícios documentados, que podem ser atingidos com alto nível de segurança. Esses benefícios incluem melhora da capacidade funcional; redução dos sintomas de isquemia miocárdica e conseqüente menor mortalidade por coronariopatia; melhoras do perfil lipídico, do peso e do controle da pressão arterial; e, em pacientes diabéticos, de tolerância à glicose. Podem ocorrer ainda melhor perfusão miocárdica, cessação do hábito de fumar e benefícios psicológicos.

\section{AGRADECIMENTOS}

Este documento foi escrito para o Colégio Americano de Medicina do Esporte por: Dr. Steven P. Van Camp (coordenador), Dr. John D. Cantwell, Dr. Gerald F. Fletcher, Dr. L. Kent Smith e Dr. Paul D. Thompson.

Este documento foi revisto para o Colégio Americano de Medicina do Esporte pelo seu Comitê de Documentos Oficiais e por: Dr. H. L. Brammel, Prof. Barry A. Franklin, Dr. G. R. Greenwell, Prof. William L. Haskell, Prof. Jeremy N. Morris e Prof. Paul Ribisl.

\section{REFERÊNCIAS}

1. Clausen JP. Circulatory adjustments to dynamic exercise and effects of physical training in normal subjects and in patients with coronary artery

Traduzido, com permissão por escrito, por:

Dr. José Kawazoe Lazzoli

Editor-Chefe da Revista Brasileira de Medicina do Esporte

Secretário da Sociedade de Medicina Desportiva do Rio de Janeiro

Professor da Universidade Federal Fluminense, Niterói-RJ

Diretor do ERGOCENTER - Instituto Petropolitano de Ergometria, Petrópolis-RJ 
disease. In: Sonnenblick EH, Lesch M, editors. Exercise and heart disease. New York: Grune \& Straton, 1977: 39-75.

2. Lazarus B, Cullinane E, Thompson PD. Comparison of the results and reproducibility of arm and leg exercise tests in men with angina pectoris. Am J Cardiol 1981;47:1075-9.

3. Bygdeman $\mathrm{S}$, Wahren J. Influence of body position on the anginal threshold during leg exercise. Eur J Clin Invest 1974;4:201-6.

4. Yasue H, Omote S, Takizawa A, Nagao M, Miwak, Tanaka S. Circadian variation of exercise capacity in patients with Prinzmetal's variant angina: role of exercise-induced coronary artery spasm. Circulation 1979; 59:938-48.

5. Hartung GH, Rangel R. Exercise training in post-myocardial infarction patients: comparison of results with high risk coronary and post-bypass patients. Arch Phys Med Rehabil 1981;62:147-50.

6. Thompson PD. The benefits and risks of exercise training in patients with chronic coronary artery disease. JAMA 1988;259:1537-40.

7. Rowell LB. Human circulation: regulation during physical stress. New York: Oxford University Press Inc., 1986:213-86.

8. Clausen JP, Trap-Jensen J. Heart rare and arterial blood pressure during exercise in patients with angina pectoris: effects of training and of nitroglycerin. Circulation 1976;53:436-42.

9. Franklin BA. Exercise training and coronary collateral circulation. Med Sci Sports Exerc 1991;23:648-53.

10. Kramsch DM, Aspen AJ, Abramowitz BM, Kreimendahl T, Hood WBJ Reduction of coronary atherosclerosis by moderate conditioning exercise in monkeys on an atherogenic diet. N Engl J Med 1981;305:1483-9.

11. Ehsani AA, Heath GW, Hagberg JM, Sobel BE, Holloszy JO. Effects of 12 months of intense exercise training on ischemic ST-segment depression in patients with coronary artery disease. Circulation 1981;6:111624.

12. Froelicher V, Jensen D, Genter F, et al. A randomized trial of exercise training in patients with coronary heart disease. JAMA 1984;252:12917.

13. Kannel WB. Contributions of the Framingham Study to the conquest of coronary artery disease. Am J Cardiol 1988;62:1109-12.

14. Rossouw JE, Lewis B, Rifkind BM. The value of lowering cholesterol after myocardial infarction. N Engl J Med 1990;323:1112-9.

15. Kallio V, Hamalainen H, Hakkila J, Luurila OJ. Reduction in sudden death by a multifactorial intervention program after acute myocardial infarction. Lancet 1979;2:1091-4.

16. Tran ZV, Brammell HL. Effects of exercise training on serum lipid and lipoprotein levels in post-M1 patients. A meta-analysis. J Cardiopulmonary Rehabil 1989;9:250-5.

17. Sparrow D, Dawber TR, Colton T. The influence of cigarette smoking on prognosis after a first myocardial infarction. J Chronic Dis 1978;31: 425-32.

18. Taylor CB, Houston Miller N, Haskell WL, DeBusk RF. Smoking cessation after acute myocardial infarction: the effects of exercise training. Addict Behav 1988;13:331-5.

19. Kannel WB, Sorlie P, Castelli WP, McGee D. Blood pressure and survival after myocardial infarction: the Framingham Study. Am J Cardiol 1980;45:326-30

20. Langford HG, Stamler J, Smollers W, Prineas RJ. All cause mortality in the hypertensive detection and follow-up program. Prog Cardiovasc Dis 1986;29:29-54.

21. Hagberg JM, Seals DR. Exercise training and hypertension. Acta Med Scand 1986;711:131-6.

22. Leon A. Patients with diabetes mellitus. In: Exercise in modern medicine. Baltimore: Williams \& Wilkins, 1989:118-45.
23. Hubert HB, Feinleib M, McNamara PM, Castelli WP. Obesity as an independent factor for cardiovascular disease: a 26-year follow-up of participants in the Framingham Heart Study. Circulation 1983;67:968-77.

24. National Obesity Consensus Conference. Ann Intern Med 1985;100:888900.

25. Oberman A, Cleary P, LaRosa JC, Hellerstein HK, Naughton J. Changes in risk factors among participants in a long-term exercise rehabilitation program. Adv Cardiol 1982;31:168-75.

26. Wilhllmsln L, Sanne H, Elmfeldt D, Grimby G, Tibblin G, Wedel H. A controlled trial of physical training after myocardial infarction. Prev Med 1975;4:491-508.

27. Ott CR, Sivarajan ES, Newton KM, et al. A controlled randomized study of early cardiac rehabilitation: the Sickness Impact Profile as an assessment tool. Heart Lung 1983;12:162-70.

28. Taylor CB, Sallis J, Needle R. The relationship of exercise and physical activity to mental health. Public Health Rep 1985;100:195-202.

29. Blumenthal JA, Emery CF, Roeski WJ. The effects of exercise training on psychosocial functioning after myocardial infarction. J Cardiopulmonary Rehabil 1988;8:183-93.

30. Oldridge NB, Guyatt G, Jones J, et al. Effects on quality of life with comprehensive rehabilitation after acute myocardial infarction. Am J Cardiol 1991;67:1084-9.

31. O'Connor GT, Buring JE, Yusaf S, Goldhaber SZ, Olmstead EM. An overview of randomized trials of rehabilitation with exercise after myocardial infarction. Circulation 1989;80:234-44.

32. Oldridge NB, Guyatt GH, Fischer ME, Rimm AA. Cardiac rehabilitation after myocardial infarction: combined experience of randomized clinical trials. JAMA 1988;260:945-50

33. Ades PA, Huang D, Weaver SO. Cardiac rehabilitation and participation predicts lower rehospitalization costs. Am Heart J 1992;123:916-21.

34. Edwards WW, Franks BD, Iyriboz Y, Dodd SL. Physiological and expense implications of PTCA rehabilitation. Med Sci Sports Exerc 1990; 22:S5.

35. American College of Sports Medicine. Guidelines for exercise testing and exercise prescription. $4^{\text {th }}$ ed. Philadelphia: Lea \& Febiger, 1991.

36. Fletcher GF, Froelicher VF, Hartley H, Haskell WL, Pollock ML. Exercise standards: a statement for health professionals from the American Heart Association. Circulation 1990;82:2286-322.

37. American College of Cardiology. Recommendations of the American College of Cardiology on cardiovascular rehabilitation. J Am Coll Cardiol 1986;7:451-3.

38. Health and Public Policy Committee, American College of Physicians. Cardiac rehabilitation services. Ann Intern Med 1988;109:671-3.

39. American College of Sports Medicine. Position stand on the recommended quantity and quality of exercise for developing and maintaining cardiorespiratory and muscular fitness in health adults. Med Sci Sports Exerc 1990;22:265-74

40. Franklin BA, Bonzheim K, Gordon S, Timmis GC. Resistance training in cardiac rehabilitation. J Cardiopulmonary Rehabil 1991;11:99-107.

41. Kelemen MH. Resistance training safety and essential guidelines for cardiac and coronary prone patients. Med Sci Sports Exerc 1989;21: 675-7.

42. Pollock ML, Gettman LR, Milesis CA, Bath MD, Durstine JL, Johnson RB. Effects of frequency and duration of training on attrition and incidence of injury. Med Sci Sports 1977;9:31-6.

43. Van Camp SP, Peterson RA. Cardiovascular complications of outpatient cardiac rehabilitation programs. JAMA 1986;256:1160-3. 